\title{
Low Loss MMI Couplers for High Performance MZI Modulators
}

\author{
D. J. Thomson, Y. Hu, G. T. Reed and J-M. Fedeli
}

\begin{abstract}
Optical splitters and combiners which can provide precise splitting with low loss, high thermal stability, large optical bandwidth, high compactness and insensitivity to fabrication tolerances are essential components for high performance MZI based optical modulators. In this paper we theoretically and experimentally investigate the MMI and the reduction in optical loss achievable through the use of linear tapers at the input and output ports. Our data shows that losses can be reduced to below $1 \mathrm{~dB} / \mathrm{MMI}$ without affecting the static extinction when employed in MZIs.
\end{abstract}

Index Terms-Silicon photonics, MMI, MZI, Optical Modulator, Taper, Low loss

\section{INTRODUCTION}

$\mathrm{H}$ igh speed silicon optical modulators have attracted significant research interest in recent years, coinciding with rapid performances enhancements. Most devices reported over the previous few years have bandwidths compatible with at least $10 \mathrm{~Gb} / \mathrm{s}$ operation. On the whole these devices consist of plasma dispersion based phase modulators commonly but not exclusively incorporated into Mach Zehnder Interferometers (MZI) structures. Large extinction ratios are obtainable using an MZI structure only if the optical power in each arm is balanced. This necessitates the use of a component which can provide precisely equal splitting (and combining) of the input light. This component is also required to cause low optical loss, have a large optical bandwidth, small footprint, high thermal stability and low sensitivity to fabrication tolerances.

A number of structures operating via different optical effects have been reported which can potentially perform this function in a silicon-on-insulator (SOI) based MZI, including directional couplers (DC), Y-splitters, star couplers and $1 \times 2$ multimode interference (MMI) structures.

Reported MZI modulators employing Y-splitters have achieved relatively low extinction ratios, in the range $10 \mathrm{~dB}$ to $16 \mathrm{~dB}$ [1-3]. Impairment of the extinction ratio can also result from an optical loss mismatch between MZI arms, however it

Manuscript received (current date), 2010. This work was supported European Community's Seventh Framework Programme (FP7/2007-2013) under grant agreement $\mathrm{n}^{\circ} 224312$ HELIOS and from the EPSRC UKSP project.

D. J. Thomson, Y. Hu and G. T. Reed are with the Advanced Technology Institute, University of Surrey, Guildford, Surrey, GU2 7XH, UK. (d.thomson@surrey.ac.uk).

J-M Fedeli is with CEA, LETI, Minatec, CEA-Grenoble, 17 rue des Martyrs, F-38054 GRENOBLE cedex 9, France. generally seems that large extinction ratios are difficult to achieve with this structure.

An MZI modulator employing a novel star coupler structure has been demonstrated [4]. The structure boasts a large optical bandwidth, small footprint and low optical loss however the static extinction ratio achieved in the MZI was only $14 \mathrm{~dB}$. Furthermore, the design features a narrow tip, complicating fabrication.

Directional couplers generally require a long coupling length for an even power split [5]. The coupling length is also sensitive to the fabrication tolerances in the waveguide dimensions and waveguide separation as well as the device temperature. Thermal sensitivity on the other hand can provide a means for tuning the splitting ratio at the cost of power consumption and device complexity.

Modulators employing splitters based upon multimode interference in a widened waveguide region (MMI) have demonstrated an extinction ratio of approximately $25 \mathrm{~dB}$ [6]. Numerous other authors have also reported MMI structures throughout the years for power splitting and combining [7-9]. MMI structures boast feature sizes typically much larger than standard DUV (Deep Ultraviolet) lithographic tool limitations easing fabrication. The splitting ratio is relatively insensitive to device temperature changes and optical throughput is only marginally affected. One drawback of this structure is the loss caused due to the modal mismatch between the MMI region and the input and output waveguides.

Some theoretical studies have investigated the reduction in loss resulting from tapering the input and output waveguides [8, 9]. The use of tapers for optical loss reduction has also been experimentally demonstrated, for example in a SOI (silicon on insulator) MMI based crossing structure [10], and in MMI couplers in other material systems [11]. Other approaches are possible for MMI optical loss reduction which include a double etch scheme [12] that has yielded an excess loss of $0.2 \mathrm{~dB}$ albeit with a more complex fabrication process. In this paper the effect of different MMI port taper widths on the loss and MZI static extinction ratio is both theoretically and experimentally investigated. Results show that as the MMI port width is increased to $1 \mu \mathrm{m}$ the optical loss drops to below $1 \mathrm{~dB}$ with no detrimental effect on the MZI static extinction ratio. The results suggest that wider tapers could also be employed which could result in further loss reductions. 


\section{MMI DESIGN}

Our design of MMI is based on the structure of $1 \times 2$ symmetric power splitter, as shown in Fig. 2. It was designed to operate at a wavelength of $1.55 \mu \mathrm{m}$ and was built upon SOI strip waveguides with a waveguide height, $\mathrm{H}$, of $220 \mathrm{~nm}$ and an input/output waveguide width, w, of $400 \mathrm{~nm}$. The width of the MMI region, W, was chosen to be $6 \mu \mathrm{m}$ to allow for compactness whilst ensuring that the output waveguides are sufficiently separated to avoid evanescent coupling from one to the other.

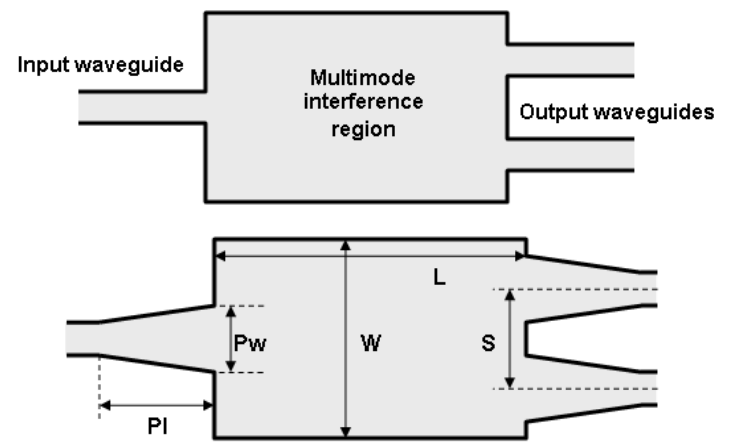

Fig. 1. MMI design without (top) and with (bottom) tapered input and output waveguides.

The length of the MMI region, $L_{\mathrm{MMI}}$, requires careful design to suitably image the light from the input port onto the output ports. In the self-imaging theory given in [13], L increases quadratically with $\mathrm{W}$ and a symmetric two-fold self-image length, by 2-D approximation, is given by:

$$
L_{M M I}=3 \pi / 8\left(\beta_{0}-\beta_{1}\right)
$$

Where $\beta_{0}$ and $\beta_{1}$ are the propagation constants of the fundamental and first order modes in the MMI region. A fully vectorial simulation tool, FIMMWAVE was used to estimate the design parameters. Firstly, the propagation constants, $\beta_{0}$ and $\beta_{1}$, in equation (1) were modelled by finding the eigenmodes of the multimode waveguide, which yields an estimated length, $L_{M M I}$ of $33.3 \mu \mathrm{m}$. Secondly, a 3-D MMI device was modelled in FIMMWAVE and it was observed from the modelled field profile (Fig. 2), that the two-fold self-image distance and the separation between the output waveguides was approximately $32 \mu \mathrm{m}$ and $3.14 \mu \mathrm{m}$ respectively. These two modelling results for the Length of the MMI match reasonably well, hence, $L=32 \mu \mathrm{m}$ and $\mathrm{S}=3.14 \mu \mathrm{m}$ were used as the design parameters.

Linear tapers were used at both input and output ports. All three tapers (as shown in Fig. 1) were identical in taper length, $P l$, and width, $P w$, at the wider ends. In order to minimise the MMI loss adiabatic tapers are required. Firstly, $P l$ is required to be sufficiently long, (here $P l$ was set as $10 \mu \mathrm{m}$ ). Secondly $P w$ should not be too wide. Fig. 4 shows the optical loss of the MMI as a function of $P w$ modelled by FIMMWAVE. An increase in MMI loss is observed when $P w$ is initially increased, however, beyond a certain critical width of approximately $500 \mathrm{~nm}$, the loss then tends to decrease with increasing $P w$. The model predicts that losses below $1 \mathrm{~dB}$ are achievable if $P w$ is widened to be $>900 \mathrm{~nm}$.

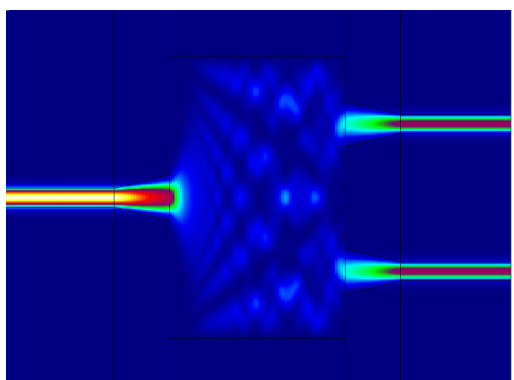

Fig. 2. MMI field profile for optimum two-fold self imaging, $L=32 \mu \mathrm{m}$, $\mathrm{W}=3.14 \mu \mathrm{m}$.

The influence of taper width on MMI loss can be explained as follows: $P w$ was kept constant for all input and output ports. The initial increase of $P w$ at the input requires even wider $P w$ at output to receive the transmitted power, until a point, where the increase of $P w$ at output matches that at the input and transmission starts to increase.

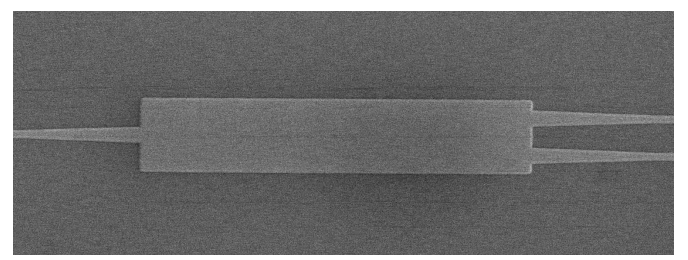

Fig. 3. SEM (Scanning Electron Microscope) image of fabricated MMI device.

The decrease in MMI loss with increasing port width has also been experimentally investigated. Device samples were fabricated on a $200 \mathrm{~mm}$ SOI wafer with $2 \mu \mathrm{m}$ buried oxide layer and 220nm silicon overlayer. DUV - 193nm lithography and $\mathrm{HBr}$ dry etching were employed to form shallow etched surface gratings for out-of-plane coupling of light to and from lensed single mode fibres. After a silica hard mask deposition, a subsequent DUV-193nm lithography and dry etching step for the hard mask and for the silicon was then performed to form the waveguides and finally an upper $\mathrm{SiO}_{2}$ cladding layer was deposited onto the wafer. An SEM image of one of the fabricated MMIs with tapered ports is shown in Fig. 3. Several MMI test structures were defined with varying port widths in $100 \mathrm{~nm}$ steps.

\section{EXPERIMENTAL AND MODELLING RESULTS}

After fabrication, devices were optically tested by measuring the throughput power of both the MMI test structures, and a standard waveguide of the same length and cross sectional dimensions. Light of wavelength $1.55 \mu \mathrm{m}$ was coupled into the devices using surface gratings. The experimental results are presented in Fig. 5. The trend of the FIMMWAVE modelling results (figure 4) are in good agreement, in that an initial increase in loss is observed with increasing port width. The loss then subsequently reduces with increasing taper width. The highlight of this result is that sub $1 \mathrm{~dB}$ losses are achieved as the 
port width is increased above $1000 \mathrm{~nm}$, close to the predicted value of $900 \mathrm{~nm}$.

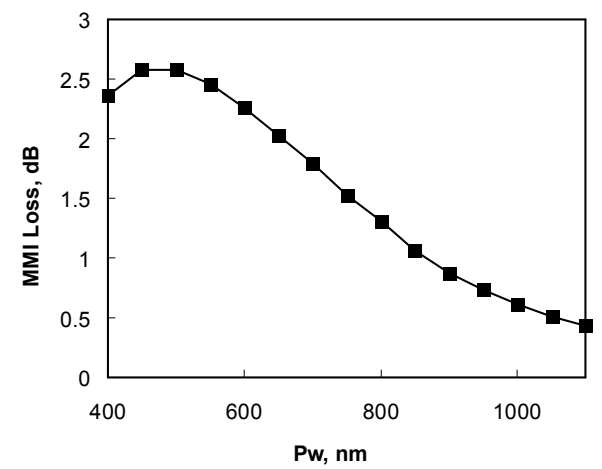

Fig. 4. Modelled MMI loss with input and output port width.

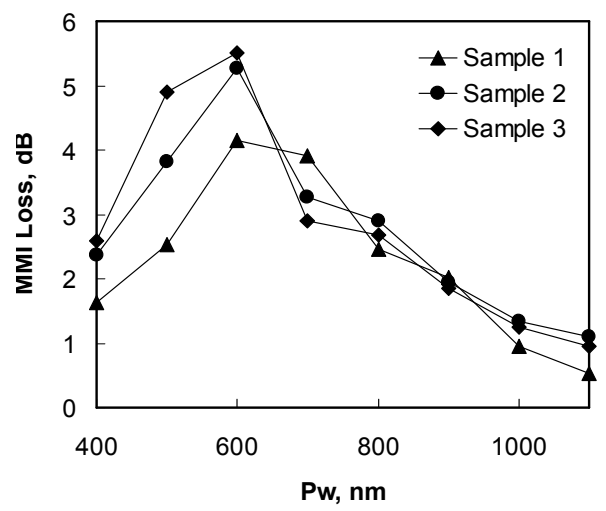

Fig. 5. Experimental MMI loss with input and output port width.

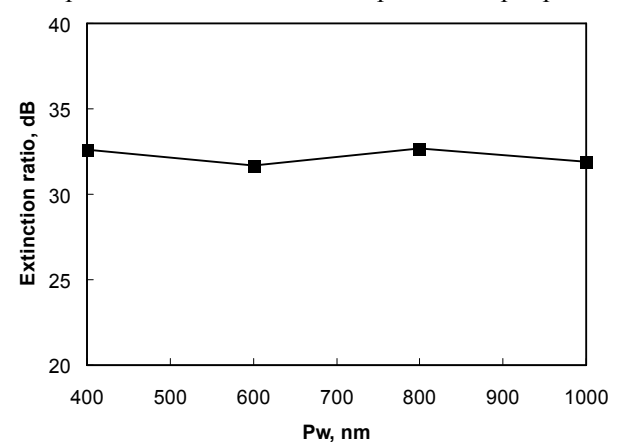

Fig. 6. MZI extinction ratio using MMI couplers with different taper widths

Four asymmetric MZI structures were also defined on the test structures each using MMI couplers with different port widths. The static extinction ratio of the MZI with increasing MMI port width is shown in Fig. 6 . These results demonstrate that not only are large static extinction ratios achieved $(>32 \mathrm{~dB})$ but that the MZI extinction ratio is largely insensitive to MMI port width over the range considered.

\section{SUMMARY}

A compact MMI structure has been investigated to provide the power splitting and combining function in MZI structures. It has been demonstrated that the MMI loss can be reduced to below $1 \mathrm{~dB}$ if the input and output powers are widened to a width of $1 \mu \mathrm{m}$ or more with minimal change of the static extinction ratio when employed in a MZI. Although the structure is not quite as compact as previously reported MMI [11], the footprint is insignificant compared to a typical MZI modulator overall (mm's), furthermore the principle reported here could be applied to small MMI with the same effect.

\section{ACKNOWLEDGMENT}

The research leading to these results has received funding from the European Community's Seventh Framework Programme (FP7/2007-2013) under grant agreement $n^{\circ} 224312$ HELIOS and from the EPSRC UKSP project.

\section{REFERENCES}

[1] A. Liu, J. Richard, L. Ling, D. Samara-Rubio, D. Rubin, O. Cohen, R. Nicolaescu, and M. Paniccia, "A high-speed silicon optical modulator based on a metal-oxide-semiconductor capacitor," Nature, vol. 427, no. 6975, pp. 615-18, Feb. 2004.

[2] T. Y. Liow, K. W. Ang, Q. Fang, J. F. Song, Y. Z. Xiong, M. B. Yu, G. Q. Lo, and D. L. Kwong, "Silicon modulators and germanium photodetectors on SOI: monolithic integration, compatibility, and performance optimization," IEEE J. Sel. Top. Quantum Electron., vol. 16, no. 1, pp. 307-315, Jan.-Feb. 2010.

[3] W. M. Green, M. J. Rooks, L. Sekaric, and Y. A. Vlasov, "Ultra-compact, low RF power, $10 \mathrm{~Gb} / \mathrm{s}$ silicon Mach-Zehnder modulator,” Opt. Express, vol. 15, no. 25, pp. 17106-17113, Dec. 2007.

[4] D. Marris-Morini, L. Vivien, J. M. Fédéli, E. Cassan, P. Lyan, and S. Laval, "Low loss and high speed silicon optical modulator based on a lateral carrier depletion structure," Opt. Express, vol. 16, no. 1, pp. 334-339, Jan. 2008.

[5] Y. J. Quan, P. Han, Q. J. Ran, F. P. Zeng, L. P. Gao, and C. H. Zhao, “A photonic wire-based directional coupler based on SOI," Opt. Communications, vol. 281, pp. 3105-3110, Feb. 2008.

[6] A. Liu, L. Liao, D. Rubin, H. Nguyen, B. Ciftcioglu, Y. Chetrit, N. Izhaky, and M. Paniccia, "High-speed optical modulation based on carrier depletion in a silicon waveguide," Opt. Express, vol. 15, no. 2, Jan. 2007.

[7] D. Dai and S. He, "Optimization of Ultracompact Polarization-Insensitive Multimode Interference Couplers Based on Si Nanowire Waveguides," IEEE Photon. Tech. Lett., vol. 18, no. 19, Oct. 2006.

[8] R. Halir, I. Molina-Fernández, A. Ortega-Moñux, J. G. Wangüemert-Pérez, D. X. Xu, P. Cheben, and S. Janz, "A design procedure for high-performance, rib-waveguide-based multimode interference couplers in silicon-on-insulator," J. Lightwave Tech., vol. 26, no. 16, pp.2928-2936, 2008.

[9] R. Halir, A. Ortega-Moñux, Í. Molina-Fernández, J. G. Wangüemert-Pérez, P. Cheben, D. X, Xu, B. Lamontagne, and S. Janz, "Compact high-performance multimode interference couplers in silicon-on-insulator," IEEE Photon. Tech. Lett., vol. 21, no. 21, pp. 1600-1602, 2009.

[10] H. Chen and A. W. Poon, "Low-Loss Multimode-Interference-Based Crossings for Silicon Wire Waveguides," IEEE Photon. Tech. Lett., vol. 21, no. 21, pp. 1600-1602, 2009.

[11] W. Bogaerts, S. Selvaraja, P. Dumon, J. Brouckaert, K. De Vos, D. Van Thourhout and R. Baets, "Silicon-on-Insulator Spectral Filters Fabricated with CMOS Technology," J. Sel. Top. Quantum Electron, Vol. 16, no. 1, pp.33-44, 2010.

[12] M. T. Hill, G. D. Khoe, and M. K. Smit, "Optimizing Imbalance and Loss in 2x2 3-dB Multimode Interference Couplers via Access Waveguide Width," Journal of Lightwave Technology, Vol. 21, No. 10, pp. 2305-2313, 2003.

[13] L. B. Soldano and E. C. M. Pennings, "Optical multi-mode interference devices based on self-imaging: principles and applications," J. Lightwave Tech., vol. 13, no. 4, pp. 615-627, 1995. 